\title{
Cerebellar Dysplastic Gangliocytoma: Case Report
}

\section{Gangliocitoma Displásico Cerebelar: Relato de Caso}

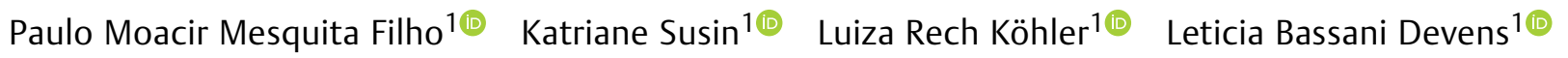

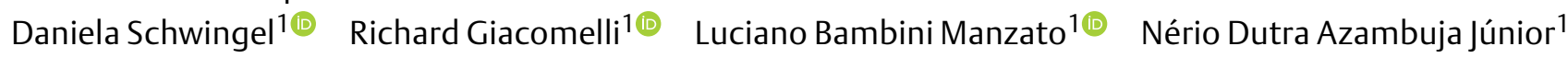 \\ ${ }^{1}$ Neurology and Neurosurgery Service, Passo Fundo, RS, Brazil \\ Address for correspondence Leticia Bassani Devens, Serviço de \\ Arq Bras Neurocir 2020;39(3):232-234. \\ Neurologia e Neurocirurgia (SNN), Passo Fundo, RS, Brazil \\ (e-mail: leticiabassanidevens@gmail.com).
}

\begin{abstract}
Keywords

- gangliocitoma

- Lhermitte-Duclos

- cerebellar tumor

- benign tumor
\end{abstract}

Lhermitte-Duclos disease (LDD), or cerebellar dysplastic gangliocytoma, is a rare type of cerebellar tumor, from unknown origin. Patients can be asymptomatic for several years, but there are usually imprecise neurological signs for long periods.

A doença de Lhermitte-Duclos (LDD), ou gangliocitoma displásico do cerebelo, é um tipo raro de tumor cerebelar, de origem desconhecida. Os pacientes podem ser assintomáticos por vários anos, mas geralmente há sinais neurológicos imprecisos por longos períodos.

\section{Introduction}

Lhermitte-Duclos disease (LDD), or cerebellar dysplastic gangliocytoma, is a rare type of cerebellar tumor of unknown origin. ${ }^{1,2}$ The first report was documented in $1920 .^{2-4}$ Patients can be asymptomatic for several years, but there are usually imprecise neurological signs for long periods. ${ }^{1,5}$ Diagnosis is made through magnetic resonance imaging (MRI) and confirmed by histopathological exam. ${ }^{1-3}$ We report the case of a 74-year-old patient, with sudden onset of symptoms, presenting this uncommon cerebellar lesion.

\section{Case Report}

A 74-year-old female patient sought medical attention due to a sudden onset of gait disturbance associated with headache and vomiting 20 days earlier. The patient had a history of tobacco use, systemic arterial hypertension, type-2 diabetes mellitus, cardiac insufficiency, schizophrenia, and a sigmoid adenocarcinoma, treated several years earlier. On neurolo- gical examination, the patient presented mild consciousness disturbance (Glasgow 14), dysmetria, and dysdiadochokinesia. An MRI was performed, which evidenced a mass lesion in the right cerebellar hemisphere, hyperintense on T2-weighted imaging and hypo intense on gadolinium-enhanced T1-weighted imaging. The lesion measured $5.8 \times 3.6 \mathrm{~cm}$ and was associated with peripheral vasogenic edema (-Fig. 1), which caused mass effect, sulci blurring among the cerebellar folia as well as compression of the cerebellarpontine cistern and fourth ventricle.

Microsurgical treatment was performed through a suboccipital craniotomy, with a near total resection of the lesion. Control computed tomography (CT) scan confirmed the extent of the resection. In the postoperative period, the patient sustained the previous deficits. Anatomo-pathological examination evidenced proliferation of round cells in the cerebellum (-Fig. 2), and immunohistochemistry was suggestive of cerebellar dysplastic gangliocytoma (World Health Organization [WHO] grade I- Lhermitte-Duclos disease), positive for Neu-N, glial fibrillary acidic protein received

August 26, 2019

accepted

March 24, 2020
DOI https://doi.org/

10.1055/s-0040-1710313. ISSN $0103-5355$.
Copyright (e) 2020 by Thieme Revinter

Publicações Ltda, Rio de Janeiro, Brazil
License terms

(®) $\Theta \circledast$ 

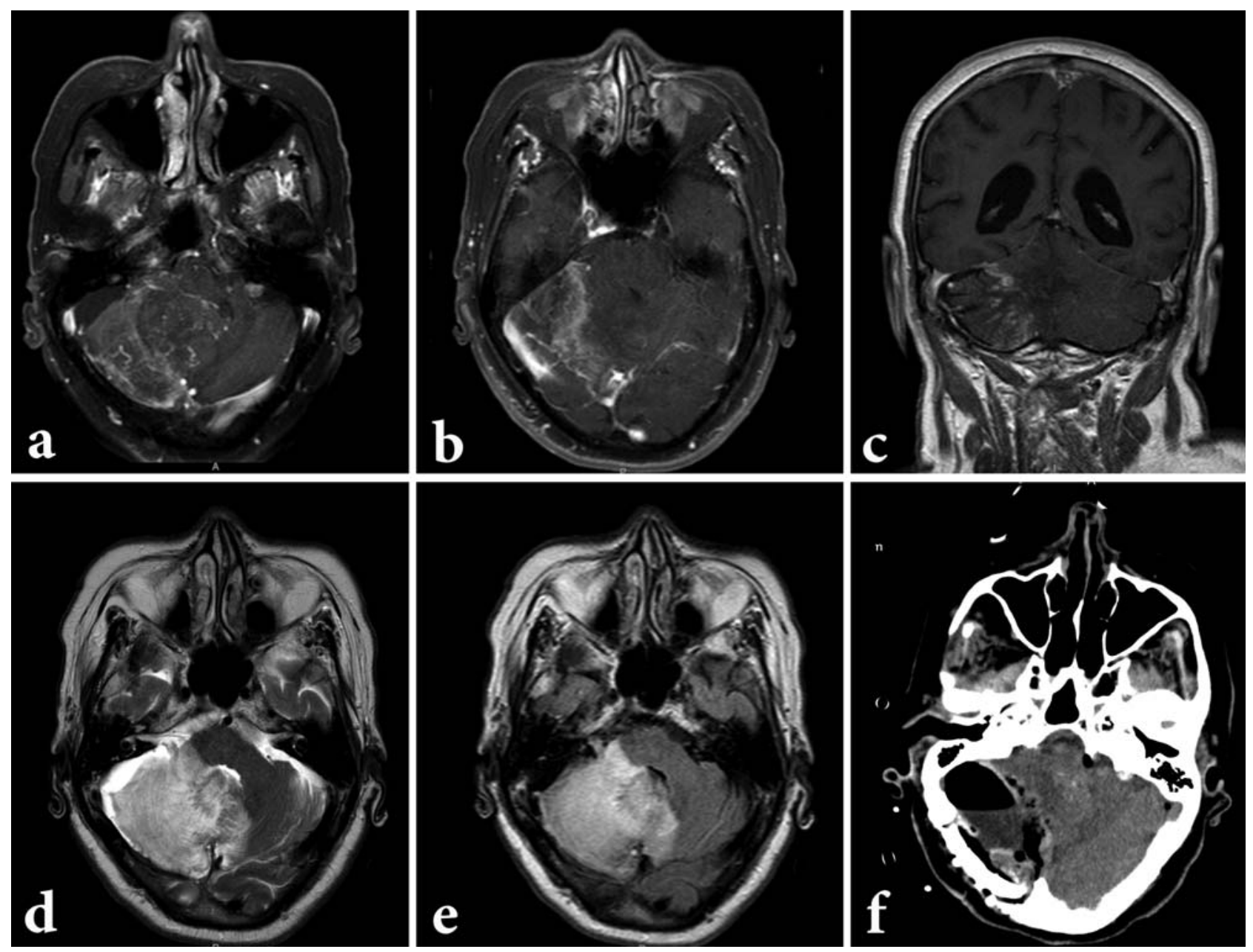

Fig. 1 (a,b) T1-weighed axial magnetic resonance imagining (MRI) with gadolinium, evidencing extensive lesion in the right cerebellar hemisphere, with imprecise limits, moderate enhancement by the contrast and mass effect, distorting the fourth ventricle. (c) T1-weighed coronal MRI. (d) T2-weighed axial MRI. (e) fluid-attenuated inversion recovery (FLAIR) axial MRI confirming the infiltrative pattern of the lesion. (f) Post-operative axial CT scan, evidencing extensive resection of the lesion.

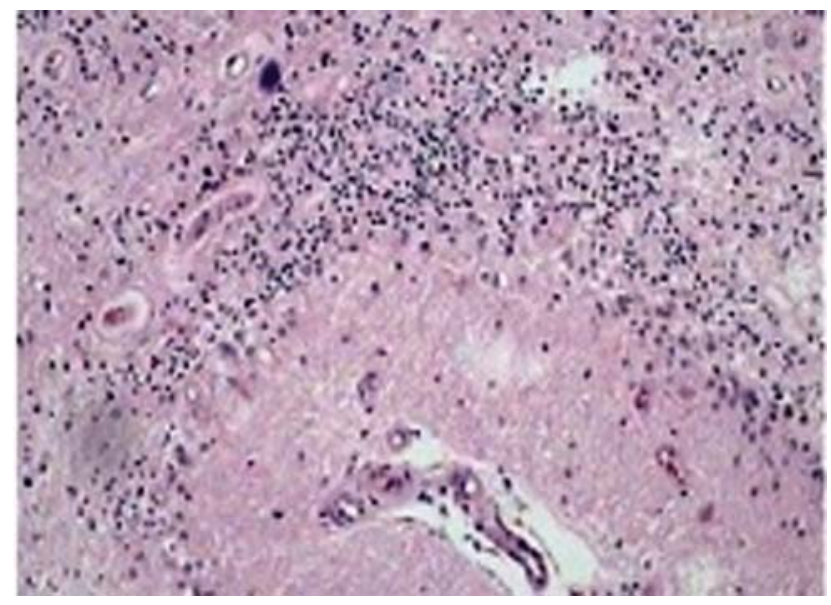

Fig. 2 Ganglionic cells with smooth atypia and single calcification focus. Hematoxylin \& Eosin stain, 50x.

(GFAP), synaptophysin and phosphatase and tensin (PTEN) antibodies.

The patient presented increase in the clinical status, with improvement in the gait, and was able to walk without assistance. The patient also present transient neuropathy of the accessory nerve. She remains in rehabilitation program to this day.

\section{Discussion}

Lhermitte-Duclos disease (LDD) is an extremely rare type of benign cerebellar tumor of unknown ethiology. ${ }^{1}$ It was initially documented in $1920 .^{3,4}$ It usually presents in young adults, with no predilection for gender or race, ${ }^{2,4,6,7}$ with a prevalence of $<1$ for $1,000,000$ patients. ${ }^{1}$ It is a lesion of the cerebellar cortex, ${ }^{3}$ characterized by loss of the normal cortex architecture and focal widening of the cerebellar folia. It is still not clear if the cerebellar dysplastic gangliocytoma is a neoplastic or hamartomatous lesion of the cortex; if neoplastic, it corresponds to a grade I lesion of the WHO classification. $^{5}$

Generally, it has an indolent and chronic course, with the possibility of acute onset of symptoms. ${ }^{3}$ It manifests with headache, visual disturbance, cerebellar dysfunction, ataxia, cranial nerve palsies, and obstructive hydrocephalus, ${ }^{2,6,7}$ mainly in the $3^{\text {rd }}$ or $4^{\text {th }}$ decade of life. ${ }^{6,7}$ Acute onset of 
neurological deficits, as in the case presented, is rarely reported. $^{5}$

Lhermitte-Duclos disease can be familiar or sporadic. ${ }^{8}$ The occurrence of associated hereditary syndromes, such as Cowden disease, has been reported. ${ }^{2,5-7}$ Cowden disease is an autosomal dominant disturbance characterized by multiple hamartomas and associated with a wide range of malignancies of the thyroid, skin, breast, intestine, and kidney. The exact correlation, nevertheless, is still uncertain. ${ }^{3}$ Molecular studies suggest a high frequency of abnormalities in the PTEN/AKT path, an important regulator of cellular growth. ${ }^{6,8}$ It is recommended that every patient diagnosed with LDD should be investigated regarding Cowden disease and mutations in PTEN. Furthermore, individuals with LDD and Cowden disease or PTEN mutation should receive continuous attention in order to prevent the associated malignancies. ${ }^{3,4,8}$

Although the confirmatory diagnosis is made through histopathological findings, the MRI can give good markers of the usual characteristics of this specific condition. Lesions are usually hypointense on T1-weighted sequence and hyperintense in T2-weighted and fluid-attenuated inversion recovery (FLAIR) sequences. ${ }^{1-3,6,8}$ Thus, widening of the cerebellar folia appears as parallel linear stria in the lesion surface. This pattern is called "tiger's stripe" or "striated cerebellum", which is characteristic of LDD. ${ }^{5,6}$ The mass lesion is circumscribed, usually restricted to one cerebellar hemisphere and has different appearance compared to the adjacent tissue. In rare cases, there is contrast enhancement, which is a finding that can represent venous proliferation of the external layers of the cerebellar cortex and prominent venous drainage. ${ }^{8}$ The computed tomography (CT) scan image characteristics consisting of hypodense areas and calcifications are unspecific to the diagnosis of LDD. ${ }^{5,7}$

The disease has classic histopathological characteristics ${ }^{3}$; the internal granular and molecular layer have diffuse enlargement, with dysplastic cells, replacing the internal molecular layer with hypermyelinization of the molecular layer. $^{1,2,6}$ There are no mitotic figures, nor necrosis. ${ }^{3}$ The Purkinje cells, as well as the white matter, are reduced or absent. ${ }^{2,5,6}$ In the immunohistochemistry studies, these cells are positive for synaptophysin., 3

The definitive treatment for this condition involves microsurgical resection of the lesion. ${ }^{1,6-8}$ Nevertheless, in asymptomatic patients, diagnosed incidentally through MRI, conservative management can be justified. ${ }^{1}$ Patients that present acute onset of symptoms, or with significant cerebellar mass effect on CT scan should be submitted to microsurgical resection of the lesion, even when the diagnosis is unclear. ${ }^{5}$ In elderly patients, partial resection can be recommended in order to reduce the mass effect and avoid surgical complications. ${ }^{2,7}$

Recurrence of the disease in the postoperative period is considered rare. ${ }^{1,6}$ Due to its association with the Cowden disease in adults, one should always rule out concomitant malignancies (particularly breast and genitourinary cancers) and perform genetic tests to diagnose Cowden disease. ${ }^{3,8}$

\section{Conclusion}

Lhermitte-Duclos disease is an extremely rare lesion of unknown etiology that can be associated with Cowden disease and mutations in the PTEN path. For this reason, if the MRI is compatible, histopathological analysis should be performed. The definitive treatment is surgical, with resection of the lesion.

\section{Conflict of Interests}

The authors have no conflict of interests to declare.

\section{References}

1 Pandey S, Sarma N. Lhermitte-Duclos Disease: A Rare Cause of Cerebellar Ataxia. Asian J Neurosurg 2017;12(04):705-706

2 Otheman Y, Aalouane R, Aarab C, Rammouz I. A case report of Lhermitte-Duclos disease revealed by psychiatric disturbances. Ann Gen Psychiatry 2017;16(01):24

3 Bhatia JK, Bhatoe HS, Vadhanan S. Lhermitte-Duclos disease: A rare entity. Med J Armed Forces India 2016;72(Suppl 1): S147-S149

4 Wang Q, Zhang S, Cheng J, Liu W, Hui X. Lhermitte-Duclos disease: Clinical study with long-term follow-up in a single institution. Clin Neurol Neurosurg 2017;162:53-58

5 Golden N, Tjokorda MG, Sri M, Niryana W, Herman S. Management of unusual dysplastic gangliocytoma of the cerebellum (Lhermitte-Duclos disease) in a developing country: Case report and review of the literature. Asian J Neurosurg 2016; 11(02):170

6 and S. P. Sugata Narayan Biswas, Partha Pratim Chakraborty, "Lhermitte-Duclos disease," 2016

7 Matsumoto H, Minami H, Yoshida Y. Lhermitte-Duclos Disease treated surgically in an elderly patient: Case Report and literature review. Turk Neurosurg 2015;25(05):783-787

8 M. Milan G Chheda, MD Patrick Y Wen, "Uncommon brain tumors," 2018 